\title{
Recent results of direct dark matter search with XMASS
}

\author{
Katsuki Hiraide ${ }^{* 1,2}$ on behalf of the XMASS Collaboration \\ ${ }^{1}$ Kamioka Observatory, Institute for Cosmic Ray Research, the University of Tokyo, \\ Higashi-Mozumi, Kamioka, Hida, Gifu, 506-1205, Japan \\ ${ }^{2}$ Kavli Institute for the Physics and Mathematics of the Universe (WPI), the University of Tokyo, \\ Kashiwa, Chiba, 277-8582, Japan \\ E-mail: hiraideakm.icrr.u-tokyo.ac.jp
}

The XMASS project is designed for multiple physics goals using the highly-purified liquid xenon scintillator detector at the Kamioka Observatory in Japan. As the first stage of the project, the detector employing approximately $830 \mathrm{~kg}$ of liquid xenon is being operated since December 2010 . In this paper, recent results of direct dark matter search in XMASS are presented.

38th International Conference on High Energy Physics 3-10 August 2016

Chicago, USA

${ }^{*}$ Speaker. 


\section{Introduction}

The XMASS project is designed to detect dark matter, ${ }^{7} \mathrm{Be} / p p$ solar neutrinos, and neutrinoless double beta decay using highly-purified liquid xenon scintillator in an ultra-low radioactivity environment [1]. As the first stage of the XMASS project, the XMASS-I detector with approximately $830 \mathrm{~kg}$ of liquid xenon was constructed [2]. It is a single-phase liquid-xenon scintillator detector located underground (2700 m water equivalent) at the Kamioka Observatory in Japan. The volume is viewed by 642 Hamamatsu R10789 photomultiplier tubes (PMTs) arranged on an $80 \mathrm{~cm}$ diameter pentakis-dodecahedron support structure. A total photocathode coverage of more than $62 \%$ is achieved. In order to shield the liquid xenon detector from external gammas, neutrons, and muon-induced backgrounds, the copper vessel was placed at the center of a $\phi 10 \mathrm{~m} \times 10.5 \mathrm{~m}$ cylindrical tank filled with pure water. The water tank is equipped with 72 Hamamatsu R3600 20inch PMTs to provide both an active muon veto and passive shielding against these backgrounds. The XMASS-I detector is being operated since December 2010. We have published results from a search for light weekly interacting massive particle (WIMP) dark matter using 6.7 live days of data [3], and a search for inelastic WIMP-nucleon scattering on ${ }^{129} \mathrm{Xe}$ using 165.9 live days of data [4].

In this paper, recent results of direct dark matter searches, a search for bosonic super-WIMPs and a search for annual modulation, are presented. We also discuss a measurement of scintillation time profile for low energy gamma-ray induced events and future prospect for pulse shape discrimination.

\section{Search for bosonic super-WIMPs [5]}

Although a WIMP dark matter is a well-motivated model and fits the cold dark matter paradigm, simulations based on this cold dark matter scenario expect a richer structure on galactic scales than those observed. Furthermore, there is so far no evidence of supersymmetric particles at the LHC, and therefore, it is important to investigate various types of dark matter candidates. These facts strengthen an interest to consider lighter and more weakly interacting particles such as superWIMPs, a warm dark matter candidate. Bosonic super-WIMPs are experimentally interesting since their absorption in a target material would deposit an energy essentially equivalent to the superWIMP's rest mass. We conducted a search for vector and pseudoscalar super-WIMPs in the mass range between 40 and $120 \mathrm{keV}$ using 165.9 live days of data collected between December 2010 and May 2012 with a restricted target mass of $41 \mathrm{~kg}$ at the central region of the detector. Figure 1 (left) shows the energy spectra of the observed events and simulated events. No significant excess above background was observed, and hence the $90 \%$ C.L. upper limits on coupling constants for pseudoscalar bosons and vector bosons were obtained as shown in Figure 1 (right). This is the first direct detection experiment exploring the vector super-WIMPs and the obtained limit for the vector super-WIMPs excludes the possibility that such particles constitute all of the dark matter. The absence of the signal also provides the most stringent direct constraint on the coupling constant of pseudoscalar dark matter to electrons. 

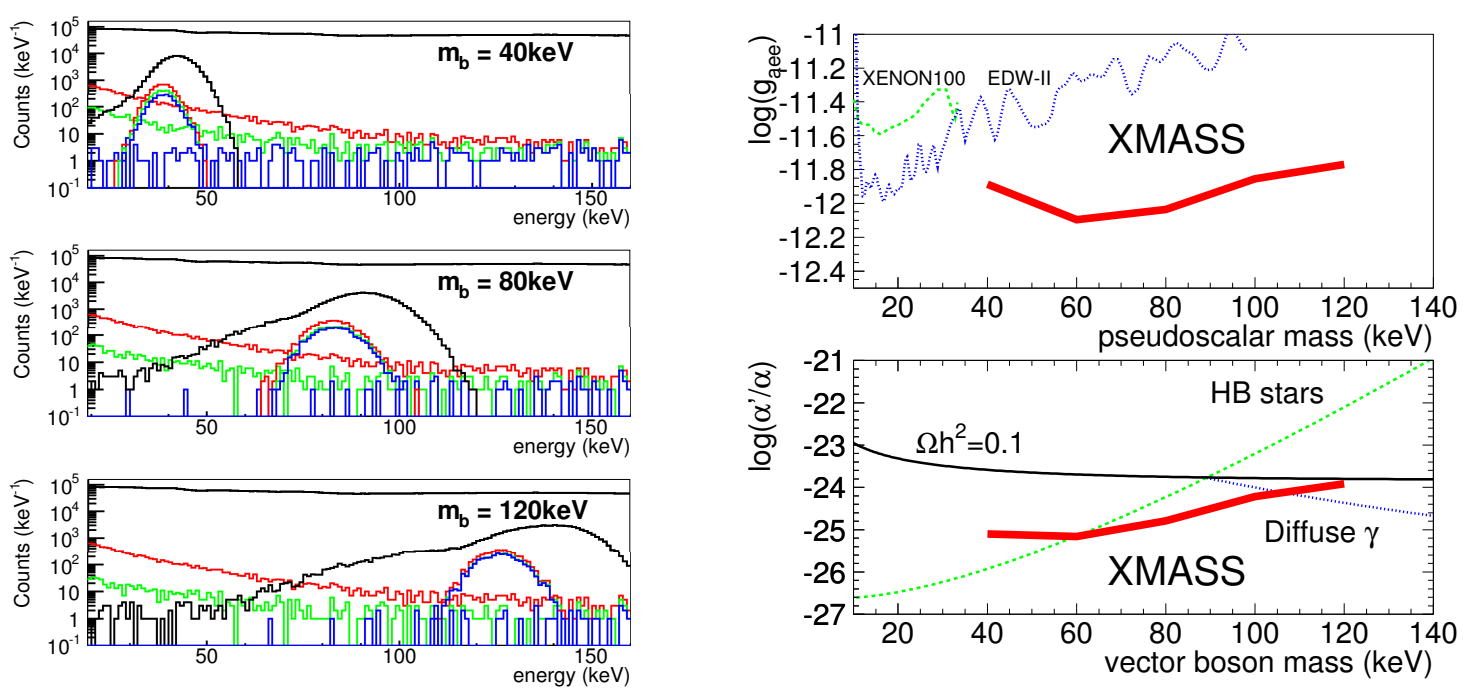

Figure 1: (Left) Energy spectra of the observed events and simulated events after each reduction step for each vector super-WIMP mass. Hatched histograms show remaining events after applying radius cut, timing cut and hit pattern cut. (Right) Obtained 90\% C.L. upper limits on coupling constants for electrons and pseudoscalar super-WIMPs (top) and for electrons and vector super-WIMPs (bottom).

\section{Search for annual modulation [6]}

The count rate of dark matter signal is expected to modulate annually due to the relative motion of the Earth around the Sun. The relative velocity of the Earth to the dark matter distribution becomes maximal in June and minimal in December. The annual modulation would be a strong signature for dark matter. We conducted a search for an annual modulation signal using 359.2 live days of data accumulated between November 2013 and March 2015. This analysis used the full $832 \mathrm{~kg}$ liquid xenon volume. The energy threshold in this analysis was $1.1 \mathrm{keV}_{\text {ee }}$ (electron equivalent), corresponding to $4.8 \mathrm{keV}_{\mathrm{nr}}$ (nuclear recoil). The data set was divided into 40 timebins with roughly 10 days of live time each. The data in each time-bin were further divided into energy-bins with a width of $0.5 \mathrm{keV}_{\mathrm{ee}}$. For the model independent analysis, the expected count rate is estimated as:

$$
R_{i, j}^{\mathrm{ex}}=\int_{t_{j}-\frac{1}{2} \Delta t_{j}}^{t_{j}+\frac{1}{2} \Delta t_{j}}\left(C_{i}+A_{i} \cos \frac{2 \pi\left(t-t_{0}\right)}{T}\right) \mathrm{d} t,
$$

where the free parameters $C_{i}$ and $A_{i}$ are the unmodulated count rate and the modulation amplitude at $i$-th energy bin, respectively. The modulation period $T$ and phase $t_{0}$ were fixed to one year and 152.5 days, respectively.

Figure 2 (left) shows the best fit amplitude as a function of energy after correcting for the efficiency. The $\pm 1 \sigma$ and $\pm 2 \sigma$ bands represent the expected sensitivity on the modulation amplitude. We evaluated the constraints on the positive and negative amplitudes separately. The $90 \%$ confidence level upper limits on the positive and negative amplitudes $a_{\text {up }}$ were calculated by the relations $\int_{0}^{a_{\mathrm{up}}} G(a) \mathrm{d} a / \int_{0}^{\infty} G(a) \mathrm{d} a=0.9$ and $\int_{a_{\mathrm{up}}}^{0} G(a) \mathrm{d} a / \int_{-\infty}^{0} G(a) \mathrm{d} a=0.9$, respectively, where $G(a)$ is the Gaussian distribution with the mean and sigma of the amplitude obtained from the fit. The XMASS experiment obtained the positive upper limits of $(1.7-3.7) \times 10^{-3}$ events $/ \mathrm{day} / \mathrm{kg} / \mathrm{keV}_{\mathrm{ee}}$ 

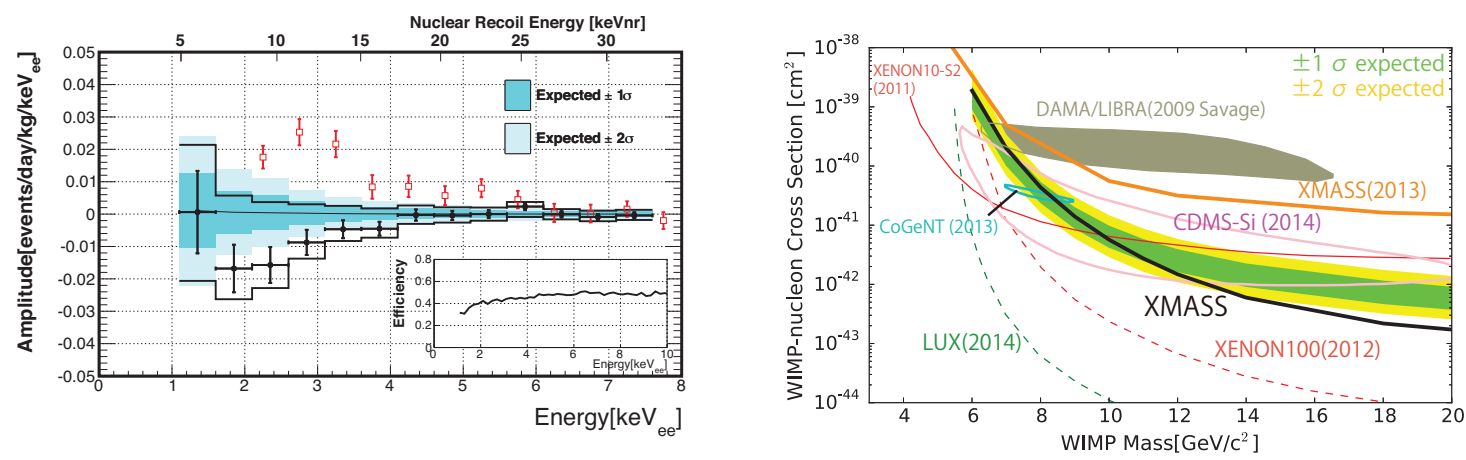

Figure 2: (Left) Observed modulation amplitude as a function of energy for the model independent analysis (circle). The solid lines represent the XMASS $90 \%$ positive (negative) upper limits on the modulation amplitude. The $\pm 1 \sigma$ and $\pm 2 \sigma$ bands represent the expected sensitivity on the amplitude. The DAMA/LIBRA result (square) is also shown. (Right) Limits on the spin-independent WIMP-nucleon cross section as a function of WIMP mass. The solid curve shows the XMASS $90 \%$ confidence level exclusion limit from the annual modulation analysis.

in the energy range between 2.0 and $3.5 \mathrm{keV}_{\text {ee }}$ and gave more stringent constraint than the modulation amplitudes observed by DAMA/LIBRA. Figure 2 (right) shows the $90 \%$ confidence level exclusion limit on the spin-independent WIMP-nucleon cross section. The result excludes the DAMA/LIBRA allowed region for the WIMP masses higher than $8 \mathrm{GeV} / c^{2}$.

\section{Measurement of scintillation time profile [7]}

The time profile of liquid xenon scintillation is important information since it could potentially be used for particle identification and vertex reconstruction. There are two scintillation processes in liquid xenon, the direct scintillation and the recombination processes. The direct scintillation process proceeds through two states, singlet and triplet excitations. The decay time constants of singlet and triplet states are a few ns and $\sim 20 \mathrm{~ns}$, respectively. The recombination process has a longer decay time constant of $\sim 30 \mathrm{~ns}$ or more. The ratio of singlet to triplet excitations as well as the recombination time depend on ionization density, and hence it can be used to discriminate between nuclear recoil events and electron events. We conducted a measurement of the scintillation time profile for low energy gamma-ray induced events using radioactive sources, ${ }^{55} \mathrm{Fe},{ }^{241} \mathrm{Am}$, and ${ }^{57}$ Co. Decay time constant was derived from a comparison of scintillation photon timing distributions between the observed data and simulated samples in order to take into account optical processes such as absorption and scattering in liquid xenon. Assuming two decay components, $\tau_{1}$ and $\tau_{2}$, the decay time constant $\tau_{2}$ increased from $27.9 \mathrm{~ns}$ to $37.0 \mathrm{~ns}$ as the gamma-ray energy increased from $5.9 \mathrm{keV}$ to $122 \mathrm{keV}$ as shown in Figure 3. The accuracy of the measurement was better than $1.5 \mathrm{~ns}$ at all energy levels. A fast decay component with $\tau_{1} \sim 2$ ns was necessary to reproduce data. Energy dependencies of $\tau_{2}$ and the fraction of the fast decay component were studied as a function of the kinetic energy of electrons induced by gamma-rays. The obtained data almost reproduced previously reported results and extended them to the lower energy region relevant to direct dark matter searches. 


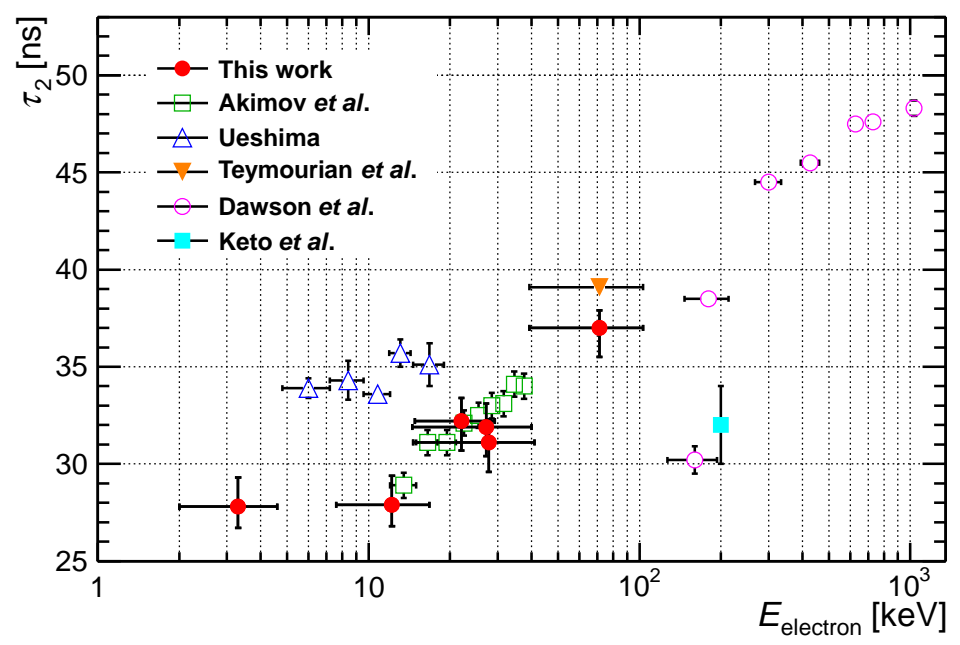

Figure 3: Decay time constant $\tau_{2}$ as a function of incident electron kinetic energy.

\section{Diversity of the XMASS experiment}

The XMASS-I detector has a high sensitivity to not only nuclear recoil but also $e / \gamma$ events, and hence achieves diversity of physics targets:

\section{- Search for solar axion [8]}

Axion would be produced in the Sun through various mechanisms such as Compton scattering of photons on electrons, $e+\gamma \rightarrow e+a$, and bremsstrahlung of axions from electrons, $e+Z \rightarrow e+a+Z$. In the liquid xenon detector, axions can be detected through the axioelectric effect, which is an analog of the photo-electric effect. XMASS has conducted a search for solar axions using 6.7 live days of data collected with lower energy threshold.

\section{- Search for two-neutrino double electron capture [9]}

Double electron capture is a rare nuclear decay process in which two orbital electrons are captured simultaneously in the same nucleus. Measurement of its two-neutrino mode would provide a new reference for the calculation of nuclear matrix elements whereas observation of its neutrinoless mode would demonstrate lepton number violation. XMASS has searched for two-neutrino double electron capture on ${ }^{124} \mathrm{Xe}$ using 132 live days of data and set the lower limit on its half-life as $T_{1 / 2}^{2 v 2 K}>4.7 \times 10^{21}$ years at 90\% CL.

\section{- Detectability of galactic supernova neutrinos [10]}

The coherent elastic neutrino-nucleus scattering $(\mathrm{CEvNS})$ is a process in which a neutrino with an energy in the order of $\mathrm{MeV}$ interacts with all nucleons in a nucleus coherently, $v+$ $A \rightarrow v+A$, resulting in a large cross section. Liquid xenon dark matter detectors are suitable for observation of galactic supernova neutrinos coherently scattered on nuclei. XMASS is expected to observe 3.5-21 events in the case of a supernova $10 \mathrm{kpc}$ away from the Earth, depending on the supernova model. 


\section{Conclusions}

The XMASS project is designed for multiple physics goals using the highly-purified liquid xenon scintillator detector at the Kamioka Observatory in Japan. As the first stage of the project, the detector employing approximately $830 \mathrm{~kg}$ of liquid xenon is being operated since December 2010. Recently, we conducted a search for vector and pseudoscalar super-WIMPs in the mass range between 40 and $120 \mathrm{keV}$ using 165.9 live days of data with a fiducial target mass of $41 \mathrm{~kg}$. This is the first direct detection experiment exploring the vector super-WIMPs and the obtained limit for the vector super-WIMPs excludes the possibility that such particles constitute all of the dark matter. We also searched for dark matter by annual modulation using 359.2 live days of data. Without assuming any specific dark matter model but including $e / \gamma$ events, the XMASS experiment obtained the $90 \%$ CL upper bounds on amplitudes that can be used to test various models. When we assume WIMP dark matter, the result excludes the DAMA/LIBRA allowed region for the WIMP masses higher than $8 \mathrm{GeV} / c^{2}$. The time profile of liquid xenon scintillation is important information for particle identification and vertex reconstruction, and hence we performed a measurement of the scintillation time profile for low energy gamma-ray induced events. In addition, the XMASS-I detector is capable of a variety of physics such as searches for solar axions, double electron capture, and galactic supernova neutrinos.

\section{References}

[1] Y. Suzuki et al., hep-ph/0008296.

[2] K. Abe et al. [XMASS Collaboration], Nucl. Instrum. Meth. A 716 (2013) 78

[3] K. Abe et al. [XMASS Collaboration], Phys. Lett. B 719 (2013) 78.

[4] H. Uchida et al. [XMASS Collaboration], Prog. Theor. Exp. Phys. 2014 (2014) 063 C01.

[5] K. Abe et al. [XMASS Collaboration], Phys. Rev. Lett. 113 (2014) 121301.

[6] K. Abe et al. [XMASS Collaboration], Phys. Lett. B 759 (2016) 272.

[7] H. Takiya et al. [XMASS Collaboration], Nucl. Instrum. Meth. A 834 (2016) 192.

[8] K. Abe et al. [XMASS Collaboration], Phys. Lett. B 724 (2013) 46.

[9] K. Abe et al. [XMASS Collaboration], Phys. Lett. B 759 (2016) 64.

[10] K. Abe et al. [XMASS Collaboration], arXiv:1604.01218 [physics.ins-det]. 